\title{
Involvement of Thermodynamic Cycle Analysis in a Concurrent Approach to Reciprocating Engine Design
}

\author{
J. Macek, M. Takáts
}

\begin{abstract}
A modularised approach to thermodynamic optimisation of new concepts of volumetric combustion engines concerning efficiency and emissions is outlined. Levels of primary analysis using a computerised general-change entropy diagram and detailed multizone, 1 to 3-D finite volume methods are distinguished. The use of inverse algorithms based on the same equations is taken into account.
\end{abstract}

Keywords: internal combustion engine, thermodynamic optimisation, simulation, configuration design.

\section{Introduction}

Ever increasing demands on efficiency and minimisation of unwanted side effects (emissions, waste heat, noise, vibrations, etc.) of engine operation may be fulfilled only if modern methods of optimisation are used as an integral part of design process. Two conditions should be respected:

- strongly limited time for development, which calls for a concurrent approach using a hierarchy of comprehensive models during all stages of development; nevertheless, these methods are fully developed for parametric design optimisation only in the sense of the terminology used in [1];

- the need for deeper configuration optimisation satisfying ever increasing competition in the prime mover market; today the reciprocating engine is king; what if it is replaced by fuel cells or by other thermal engines (including steam engines) in the future?

The base for every parametric optimisation is a properly calibrated model of sufficient breadth (i.e., comprehensiveness of the description of the engine and accessories system, or even involvement of an engine in the vehicle system) and depth (i.e., involvement of phenomena and independent co-ordinates).

In all usable cases, the knowledge acquired and the computer power available limit the depth. Therefore the universally valid laws of conservation have to be supplemented by empirical closures. They have a different range of validity, starting with an equation of state, Fourier's or Fick's laws and ending with turbulence and chemical kinetic models or even purely empirical correcting functions (e.g., rate-of-heat-release function, $\boldsymbol{R O H R}$, heat transfer coefficient correlations) or correcting factors (e.g., loss or discharge coefficients). Changing the closures, the model should be calibrated for the considered class of engines or optimisation tasks. The structure of a model must be transparent, otherwise the calibration is dubious and the results are unreliable. In this case "less means more" very often. Accumulation of too many closures (especially if they come from different research sources) tends to unreliability and fuzziness.

Concerning configuration design the concept under consideration should be divided into simple sub-systems and new combinations of them should be studied. This procedure gives an apparently countable but very high number of variants that must be sorted before detailed calculations are made. The description of system elements consists in algorithms that are already developed. Nevertheless, it is not suitable to apply them for all possible cases because it consumes too much time. The preliminary sort procedure must be applied. It is to some extent heuristic. On a higher level, some new elementary sub-systems may even be amended. Then the process is also heuristic at a higher level.

The aim of this paper is to present an analysis of thermodynamic model division into elements and possibilities of acquiring simple first-approximation sorting aids for further synthesis, to show some unavoidable associations with engine and vehicle structure dynamics, and to stress the results of inverted algorithms for model calibration.

\section{Ways to improve the engine concept}

Analyses of combustion have shown that a compromise between Carnot limits of cycle efficiency and temperature effect on $\mathrm{NO}_{\mathrm{x}}$ formation should be sought for. Homogeneous combustion without stepwise heating and compression of the burnt zones would be desirable if feasible, if firing pressure is not too high and if a lean mixture causing low specific power is used. Removal of the impacts caused by a limited expansion ratio would open another well-known possibility of higher efficiency followed, however, by decreased mechanical efficiency and higher cooling losses due to the low specific power of an engine. Conversely, engine downsizing by turbocharging is valuable from the standpoint of specific power, i.e., heat cooling losses and mechanical efficiency but in most cases it requires higher piston work during gas exchange and very high firing or compression pressures. Thus, most measures have an ambiguous impact if multi-criterion optimisation is 
taken into account. Moreover, all of them result in increased complexity of engine design and the need for better control algorithms, preferentially with adaptive and predictive capabilities. Therefore, improved predictive tools are essential and interaction between thermodynamics and mechanical efficiency must be taken into consideration. An example of a design network involving these factors is presented in [1].

\section{Tools of standard and configuration thermodynamic analysis}

Thermodynamic simulation is based on the laws of conservation, constitutive equations and empirical closures based on goal-directed experiments. The hierarchy of models covers a wide range from an idealised thermodynamic cycle over 0D, 1D and zone approaches [2] to comprehensive system models with 3D CFD simulation. A modular approach used very widely by Gamma Technologies (see, e.g., in [2]) has been further elaborated to 3-D in [3], [4]. The breadth of the models is indirectly proportional to their depth, of course, so that all of the models mentioned may play their proper role in engine cycle analysis.

The layout of a current state-of-the-art 1-D model is presented in Fig. 1, together with examples of new engine concepts not included in a standard model - [5], [6]. A parametric optimisation - [1] - is solved fully by this model, today often with automated procedures - [2] and interactions to engine gear mechanics - e.g., [7], [8]. This link is of utmost importance not only because of the impact of pressure-volume dependence on mechanical losses (especially using high compression ratios and boost pressure levels for turbocharged engines) but due to power input for accessories, especially injection equipment (ultra high-pressure direct injection, common rail injection) or variable valve gear timing with advanced electric actuators of rather limited mechanical efficiency.
However, new configuration design tools should be sought for because conventional tools are not adapted to the flexibility required by changes in volumetric engine layout. The general approach used is to divide complex models into elementary parts and to allow for the construction of an arbitrary set of these modules to a new concept. A "thermodynamic structure kit" has to be developed for these purposes. Using procedures and data (especially substance properties), a thermodynamic structure kit may be used even for formulating the inverse algorithms suitable for evaluating experiments and calibration of models - [9]. Moreover, an initial sorting-out tool should be provided.

\section{Simple configuration design tool}

Starting with the last issue, a versatile simplest model suitable not only for current tasks has been developed by computerising the old entropy (T-s) diagram approach. It is based on general reversible polytropic changes for the high-pressure part of a cycle where the mass of gas inside a cylinder is constant. The model contains in the most general manner (see, Fig. 3 left):

- polytropic compression (1-2), which can be divided (1-12 and 12-2) into heated (the only case in the figure) and cooled parts, if necessary, taking internal heat regeneration from hot components (if present) into account;

- constant-volume (isochoric) heat supply caused by regenerated heat at the end of the compression stroke (2-22, if present); this part ends when the unsteady surface temperature of surrounding walls is achieved;

- constant-volume heat supply by combustion (22-23), optionally with simultaneous heat loss (covered by fuel heat supply) to engine components with a temperature lower than that of the gas;

- constant pressure (isobaric) combustion (23-3), optionally amended as in the two previous issues depending on the gas temperature reached after the constant-volume phase;
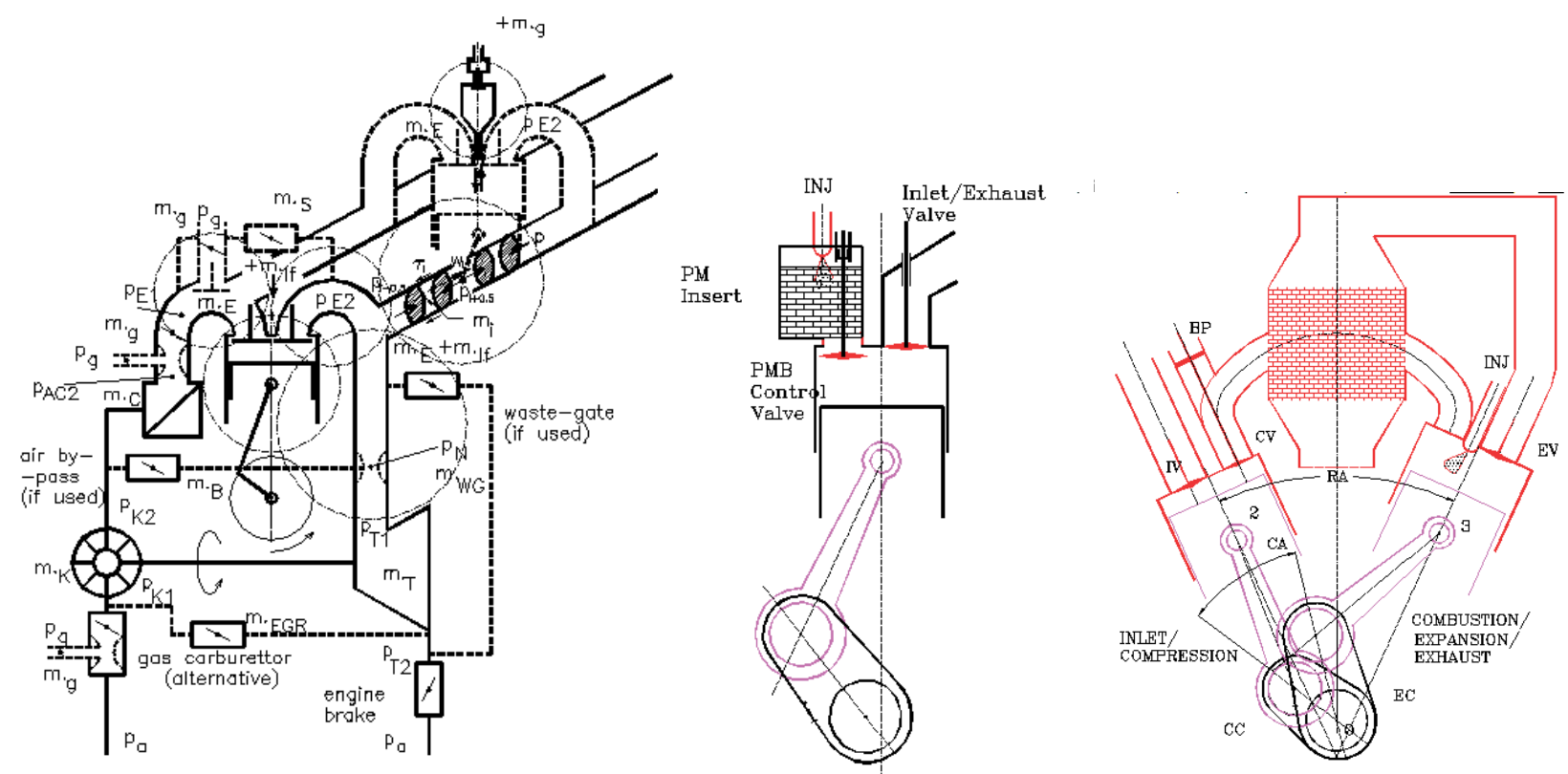

Fig. 1: Layout of a standard 1-D engine model and examples of unconventional reciprocating engines with porous medium combustion (middle) - [5] or exhaust gas heat regeneration (right) - [6] 
- polytropic combustion (heated expansion due to afterburning) (3-334), optionally amended as in the previous issues, in most cases an isothermal combustion gives a suitable approximation;

- polytropic expansion (34-4) (usually cooled) optionally amended as in the previous issue (34-344 and 344-4); this can again be divided into cooled (high gas temperature) and heated parts (the case of gas temperature below the surface temperature is presented in the figure);

- constant-volume heat rejection substituting the real gas expansion to an exhaust system; the first part of it (4-55) concerns the part of the heat stored in a hot component heat capacity, e.g., the next change 2-22; these changes can be applied only if suitable engine lay-out is used;

- constant-volume heat rejection, in reality provided by the exhaust process - gas expansion and scavenging (55-6, which should be identical with 55-1).

This model uses realistic heat capacities dependent on temperature and gas composition. Heat supply from fuel chemical energy is calculated in 3 or 4 steps (i.e., 2-34), taking overall cooling losses determined by experiments or by more detailed cycle simulation into account, so detailed model calibration is provided, avoiding the very rough standard approach an using overall correcting coefficient only. On the other hand, all limiting values are maintained at a prescribed level during calculation, using nested iterations. Irreversible changes in an exhaust system, turbine and compressor are modelled in a simplified manner for turbocharged engines to take into account changes in energy transfer to the turbine caused by high-pressure cycle changes.

The model is precise if all limiting values and the engine cooling loss are known - Fig. 2. Some examples of results of cycle optimisation are presented in Fig. 3. The importance of this model consists in fact that not only quantitative results are obtained during optimisation but that the shape of the T-s diagram generates instructions for improvements. Generally speaking, all measures enlarging the "entropy length" of the diagram are harmful if no internal heat regeneration at a suitable temperature difference is used. This approach provides therefore for parameters and fixes - [1] simultaneously. Nevertheless, a more profound analysis is necessary after the first reasoning has been finished. The elements of a "thermodynamic structure kit" are therefore described now.

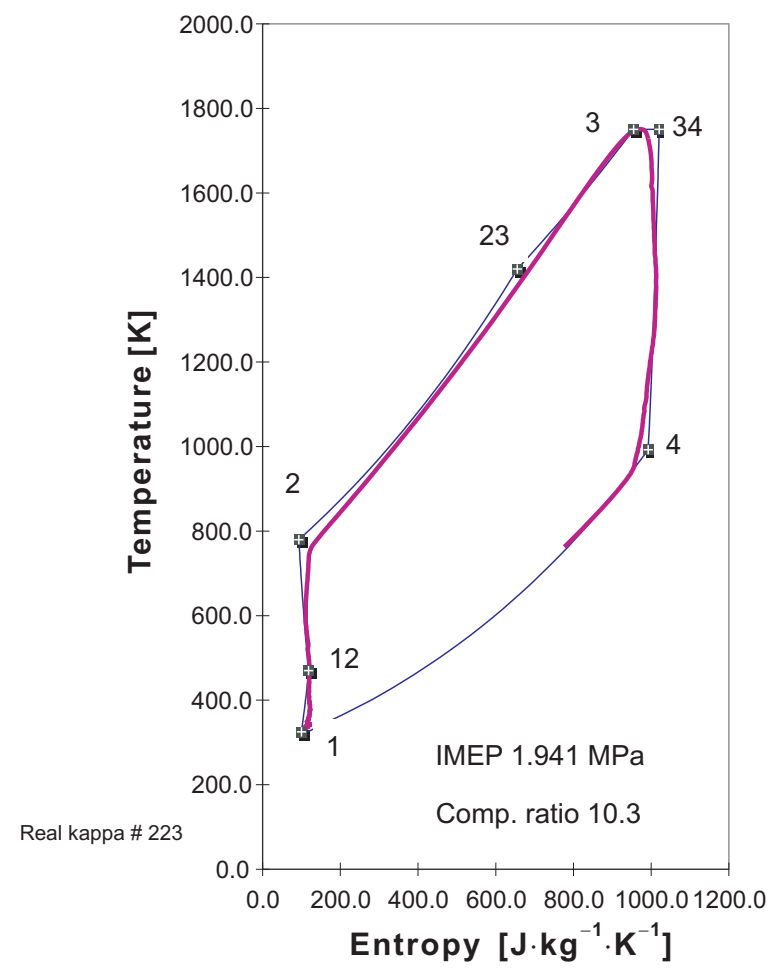

Fig. 2: Entropy diagram of a real cycle with a lean mixture and an ignition amplifier (a pre-chamber), limited pressure and temperature, low NOx emission, high thermal efficiency. A comparison of an idealised cycle and a real cycle (error in thermal efficiency of $0.5 \%$ )
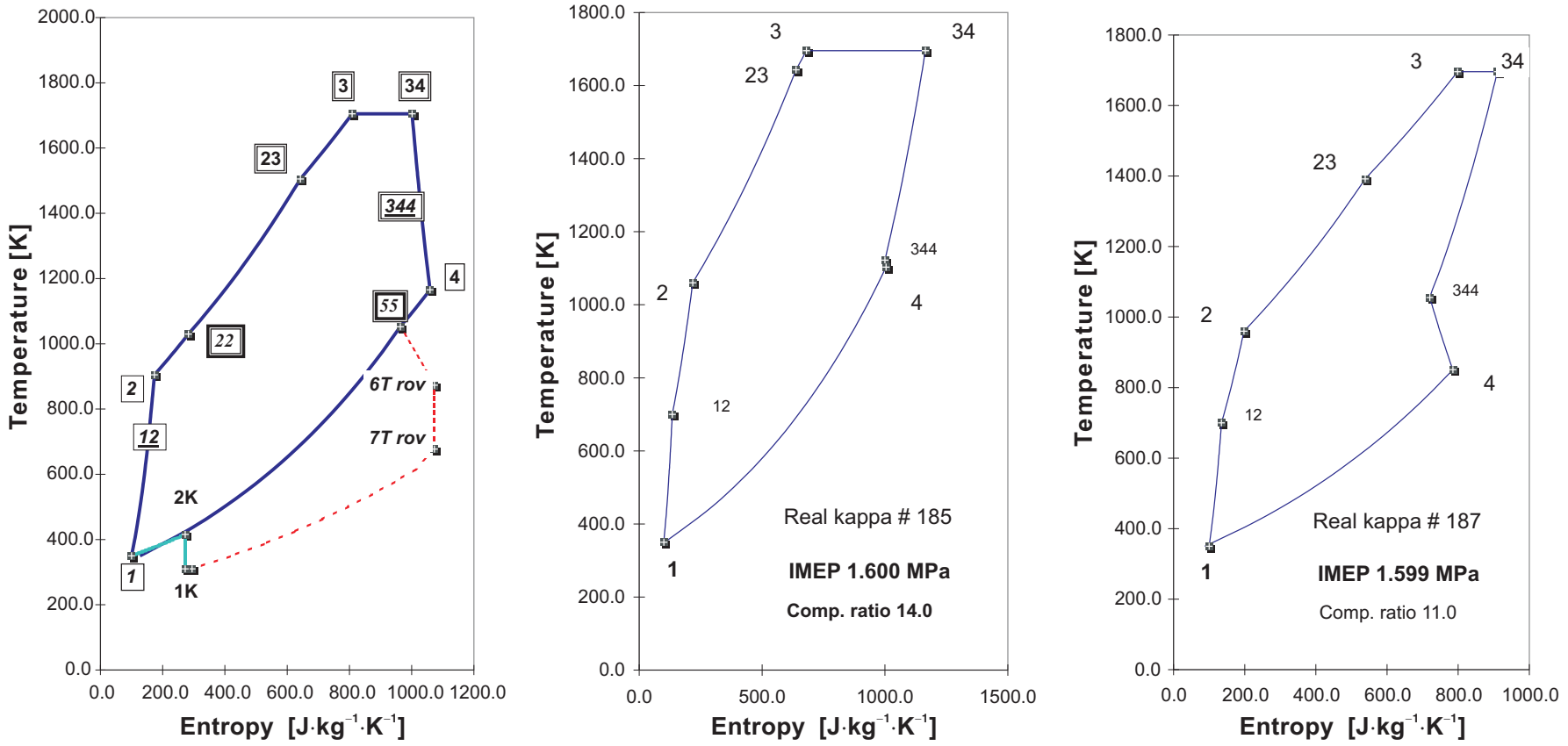

Fig. 3: Entropy diagram of a general cycle with heat regeneration and a turbocharger and examples of some interesting cycles with limited pressure and temperature and comparatively high thermal efficiency if heat regeneration between 34-344 and 12-2(-22) is used 


\section{Basic "Boundary condition" equations}

Before the concrete appearance of the conservation laws for a modular model is presented the structure of the model and its boundary conditions must be described. In reality, partial differential equations describe the continuum mechanics inside an engine. The model represents a real continuum, using concentrated parameters in finite volumes $(\boldsymbol{F V})$ and in the case of necessity dividing manifolds into parts. Therefore it can be described by the system of ordinary differential equations (ODE). In this case the boundary conditions of the original set of partial differential equations are transformed to algebraic linking equations that occur in the right-hand side vector of an ODE set.

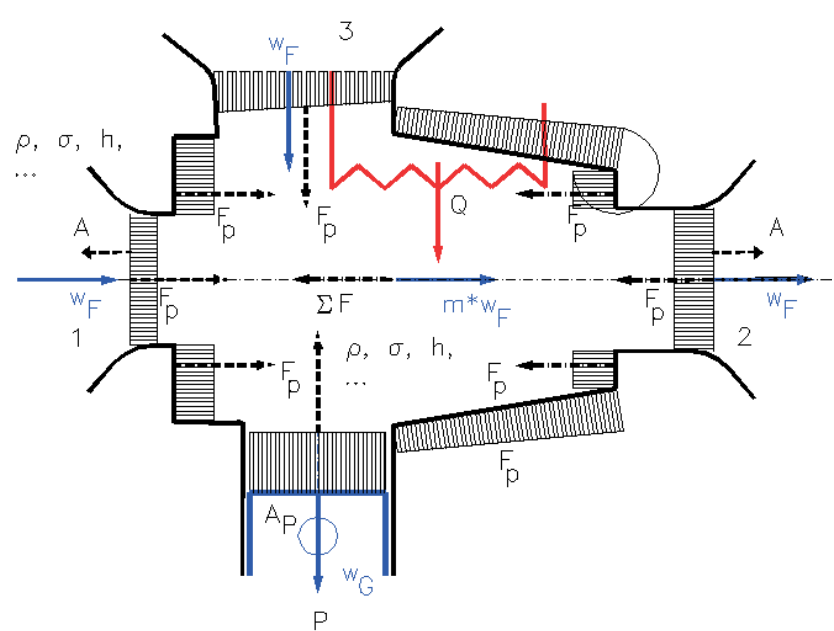

Fig. 4: Finite volume as an element of a modular simulation tool

The model consists of the complicated structure of such finite volumes in the amount of $m$ with 1-D or sometimes 0-D unsteady flows connected mutually by throttling devices (ports, valves, nozzles, diffusers, etc.) and featuring moveable walls in general. The structure itself can be described in a finite-volume index $m^{*} m$ matrix, where linked finite volumes are stated by non-zero terms. In the case of a serial model structure the index matrix is tri-diagonal. It is used for defining the extent for sum operations over neighbouring volume fluxes in the resulting ODE set.

An example of a general model element (finite volume) with a 1-D co-ordinate in its axis, mass fluxes, acting pressure forces, heat source and power sink (due to a moveable wall) is presented in Fig. 4.

The key terms are those describing volumetric fluxes. The volume flux is oriented by the result of the scalar product, using velocity vectors $w_{\mathrm{F}}$ and outer-normal oriented surface vectors $A$ at the linking surfaces. It is not associated with up-wind procedure described further only if the fluid is incompressible. Since the compressibility of gases may not be neglected for typical flows of reciprocating engines (even if they are in most cases subsonic) the volumetric flux must be reduced to the upstream gas density. Nevertheless, using the volumetric flux remains suitable for versatile flux formulae, and therefore it will be used in the following. The sign of velocity (i.e., the upstream and downstream $F V$ ) is obtained basically by comparing the static pressures in neighbouring volumes (a corrective procedure taking inertia into account is outlined further). Thus using index $A$ for the effective throat cross-section (including flow contraction), $\boldsymbol{j}$ for the FV under consideration and $i$ for the neighbouring ones, the volumetric flux $V$ from $i$ to $j$ yields

$V_{j, i}=-\vec{w}_{j, i} \cdot \vec{A}_{j, i} \cdot \frac{\rho_{\mathrm{A}}}{\rho_{\text {up } j, i}}$;

$\boldsymbol{\Phi}_{j, i}=V_{j, i} \cdot\left(\phi_{i} \cdot \rho_{i} \frac{1+\operatorname{sign} V_{j, i}}{2}+\phi_{j} \cdot \rho_{j} \frac{1-\operatorname{sign} V_{j, i}}{2}\right)$.

The second equation easily determines all fluxes $\Phi$ in consideration (using mass-specific $\varphi$ value for specie, momentum, and energy), which are calculated using up-winding according to the sign of $V$. The up-winding terms "inside" and "out-of" - fractions containing the sign function - are indicated as $\gamma_{j, i}, \delta_{j, i}$ respectively in the following.

Although formulae for compressible flow volumetric or mass flux seem to be well known, two modifications should be involved for the current case - [10]. In most cases, the port and throat length (B.C.L.) is not negligible in comparison with the $F V$ length, but it cannot be reduced simply to the $F V$ itself. The steady-flow energy equation ("total enthalpy conservation") can be supplemented by an approximation of an unsteady term expressing the work of local inertia forces in a similar manner as in the case of the unsteady term in the Bernoulli equation. It yields for correcting term $I$ using reduction of active gas column lengths $L_{\mathrm{n}}$ ( $K$ being index of total state, up means upstream position)

$$
\begin{aligned}
& h_{\text {Kup }}=h_{A}+\frac{w_{A}^{2}}{2}+I \text {; } \\
& I=\int_{B . C . L .} \frac{\partial w}{\partial t} \mathrm{~d} L=\frac{\partial w_{A}}{\partial t} \sum_{n} L_{\mathrm{n}} \cdot \frac{A_{j, i}}{A_{n}} ; \\
& \frac{\partial w_{A}}{\partial t}=K \frac{\partial}{\partial t}\left(\sqrt{p_{K u p}-p_{A}}\right)=\frac{K}{2} \cdot \frac{\frac{\mathrm{d} p_{K u p}}{\mathrm{~d} t}-\frac{\mathrm{d} p_{A}}{\mathrm{~d} t}}{\sqrt{p_{K u p}-p_{A}}}
\end{aligned}
$$

This assumes negligible density changes due to inertia effects. The last expression uses Bernoulli's approximation of flow rate, which simplifies the derivative of the pressure function. The factor $K$ can be solved iteratively comparing precise and Bernoulli solutions, which is quite suitable because of the iterative essence of the whole procedure, as will be demonstrated further in the equation (3).

The second remark concerns complex influence of the velocity loss coefficient $\varphi$. It is widely used for ideal (isentropic) velocity correction (see the right-side expression in the following formulae) but without taking into consideration other impacts of kinetic energy dissipation, namely on the critical pressure ratio and throat density. The more precise expressions yield

$$
\begin{aligned}
& \varepsilon=\max \left(\frac{p_{A}}{p_{K u p}} ; \varepsilon^{*}=\left[1-\frac{\kappa-1}{\varphi^{2}(\kappa+1)}\right]^{\frac{\kappa}{\kappa-1}}\right) ; \\
& \frac{\rho_{A}}{\rho_{K, u p}}=\frac{\varepsilon}{1-\varphi^{2}\left(1-\varepsilon^{\frac{\kappa-1}{\kappa}}\right)} ;
\end{aligned}
$$




$$
w_{j, i}=\varphi \sqrt{\frac{2 r \kappa}{\kappa-1} \cdot T_{K, u p} \cdot\left(1-\varepsilon^{\frac{\kappa-1}{\kappa}}\right)-I} .
$$

An inertia correction term is included. With it, the limits for the subsonic region must be tested iteratively, using the pressure ratio limit as the first approximation and checking the resulting flow velocity against the local sonic velocity (this is calculated involving also dissipation of kinetic energy, using a temperature term in the denominator of the density expression). Problems may occur if pressure difference is very near to zero, paradoxically. These cases must be treated carefully.

The deduced formulae are used in linking terms of the convective fluxes involved in the right-hand sides of all conservation equations (6), (10), (11). Values with index Kup have to be substituted by the concrete total state upstream of the throttling device, $A$ means static pressure downstream of it. In most cases the latter means static pressure in the following $F V$, but in some cases it is worthwhile to differentiate between them if the diffuser effect downstream of a bottleneck takes place. It can be computed even in dissipative cases of Borda loss.

\section{Conservation equations for a FV}

The general form of conservation equations is used in accordance with the literature - see, e.g., [3]. A column vector of $s$ species is designated by $\left\{_{\mathrm{s}}\right\}$, the weighted sums of mass-specific quantities are calculated using the scalar product written in matrix notation as transposed (row) vector times column one, i.e., $\left\{\left\{_{\mathrm{s}}\right\}^{\mathrm{T}} \cdot\left\{_{\mathrm{s}}\right\} ; \sigma\right.$ stands for mass fractions, $\rho$ for density, index $\mathrm{CH}$ chemical reactions (primarily combustion). All chemistry is described for $r$ reactions by transformation stoichiometric matrix $\|C\|-$ Tab. 1 and [9]).

The chemical transformation of species yields for concentration changes, using the stoichiometric transformation matrix together with Guldberg-Waage-Arrhenius laws (5)

$$
\begin{aligned}
& V \frac{\mathrm{d}\left\{{ }_{\mathrm{s}} c\right\}_{\text {chem }}}{\mathrm{d} t}=\|C\|_{\mathrm{S}} *_{\mathrm{r}} \frac{\mathrm{d}\left\{{ }_{\mathrm{r}} m\right\}}{\mathrm{d} t}=\|C\|_{\mathrm{S} * \mathrm{r}} \cdot V \cdot \frac{\mathrm{d}\left\{{ }_{\mathrm{r}} c\right\}}{\mathrm{d} t}, \\
& \frac{\mathrm{d}\left\{{ }_{\mathrm{r}} c\right\}}{\mathrm{d} t}=\left[{ }_{\mathrm{r}} K \cdot T^{-\mathrm{b}} \cdot \mathrm{e}^{-\frac{\mathrm{E}}{\mathrm{R} \cdot \mathrm{T}}} \cdot c_{\mathrm{X}}^{\mathrm{X}} \cdot c_{\mathrm{Y}}^{\mathrm{y}} \cdot c_{\mathrm{Z}}^{\mathrm{Z}}\right] .
\end{aligned}
$$

Here $K$ stands for a reaction constant (the individual constants create a vector $\left.\left\{{ }_{r} K\right\}\right)$ and $E / R$ is an individual activation energy for a certain reaction. They are together with the exponents $b, x, y, z$ determined from experiments for the reactions described by columns of $\|C\|-$ Tab. 1 .

Liquid fuel evaporation may be taken into consideration in a similar way; up-wind coefficients $\gamma, \delta$ have already been mentioned. Then the conservation of species yields

$$
\begin{aligned}
& \frac{\mathrm{d}\left\{{ }_{\mathrm{s}} m\right\}_{j}}{\mathrm{~d} t}=\sum_{i=N_{j}}\left[V_{j, i}\left(\rho_{i}\left\{{ }_{s} \sigma\right\}_{i} \cdot \gamma_{j, i}+\rho_{j}\left\{{ }_{s} \sigma\right\}_{j} \cdot \delta_{j, i}\right)\right]+ \\
& +\frac{\mathrm{d}\left\{{ }_{\mathrm{s}} m\right\}_{C H j}}{\mathrm{~d} t} ; \\
& \frac{\mathrm{d}\left\{{ }_{\mathrm{s}} m\right\}_{C H j}}{\mathrm{~d} t}=\left\|_{\mathrm{r}{ }_{\mathrm{s}}} C\right\| \cdot \frac{\mathrm{d}\left\{{ }_{\mathrm{r}} m\right\}_{j}}{\mathrm{~d} t} .
\end{aligned}
$$

Volumetric fluxes associate this equation (6) with energy equations since they include pressures both inside zone (FV) $j$ and in neighbouring FVs. In the neighbourhood there may be fictive zones as well (e.g., plenum with steady state pressure and temperature).

Before other conservation equations are presented the equation of state, which supplemented the ODEs set to a solvable system, has to be transformed to a suitable form.

The thermodynamic state equation can be written in the form $p=\mathrm{f}\left(T,\left\{{ }_{\mathrm{s}} m\right\}^{\mathrm{T}}, V\right)$ after molar-specific quantities are simply recalculated in mass-specific quantities. In the case of real gas mixtures, the empirical coefficients have to determined.

\begin{tabular}{|c|c|c|c|c|c|c|c|c|c|}
\hline $\begin{array}{c}\text { Reaction main component } \\
/ / \\
\text { Specie: }\end{array}$ & $\mathbf{H}_{2}$ & $\mathrm{H}_{2} \mathrm{O}$ & CO & $\mathrm{CO}_{2}$ & $\mathbf{C}_{8} \mathbf{H}_{18}$ & $\mathbf{N}_{2}$ & $\mathbf{N}$ & NO & NO \\
\hline $\mathrm{O}_{2}$ & $-16 / 2$ & $+16 / 18$ & $-16 / 28$ & $+16 / 44$ & $-400 / 114$ & & $-32 / 14$ & & $+32 / 30$ \\
\hline $\mathbf{H}_{2}$ & $-2 / 2$ & $2 / 18$ & & & & & & & \\
\hline $\mathrm{H}_{2} \mathrm{O}$ & $18 / 2$ & $-18 / 18$ & & & $162 / 114$ & & & & \\
\hline CO & & & $-28 / 28$ & $28 / 44$ & & & & & \\
\hline $\mathrm{CO}_{2}$ & & & $44 / 28$ & $-44 / 44$ & $352 / 114$ & & & & \\
\hline $\mathrm{C}_{8} \mathrm{H}_{18}$ & & & & & $-114 / 114$ & & & & \\
\hline $\mathbf{N}_{2}$ & & & & & & $-28 / 28$ & $-14 / 14$ & $28 / 30$ & \\
\hline $\mathbf{N}$ & & & & & & $14 / 28$ & & $-14 / 30$ & $14 / 30$ \\
\hline NO & & & & & & $30 / 28$ & $30 / 14$ & $-30 / 30$ & $-30 / 30$ \\
\hline O. & & & & & & $-16 / 28$ & $16 / 14$ & $16 / 30$ & $-16 / 30$ \\
\hline
\end{tabular}
The correlations for it are available (e.g., nonlinear molar-weighted sums in the case of a suitable B-W-R equation of state). Thus,

Table 1: An example of a part of the transformation matrix $|\mathbf{C}|$ for some combustion products of octane and nitrogen oxides formation according to Zeldovitch. Columns describe reactions designated by the main reaction component. 


$$
\begin{aligned}
& p=p\left(T, V,\left\{{ }_{\mathrm{s}} m\right\}\right) ; \\
& \frac{\mathrm{d} p_{j}}{\mathrm{~d} t}=\frac{\partial p}{\partial T} \cdot \frac{\mathrm{d} T_{j}}{\mathrm{~d} t}+\frac{\partial p}{\partial V} \cdot \frac{\mathrm{d} V_{j}}{\mathrm{~d} t}+\sum \frac{\partial p}{\partial m_{i}} \cdot \frac{\mathrm{d} m_{i, j}}{\mathrm{~d} t} \\
& \frac{\mathrm{d} T_{j}}{\mathrm{~d} t}=\frac{\frac{\mathrm{d} p_{j}}{\mathrm{~d} t}-\frac{\partial p}{\partial V} \cdot \frac{\mathrm{d} V_{j}}{\mathrm{~d} t}-\sum_{i}^{s} \frac{\partial p}{\partial m_{i}} \cdot \frac{\mathrm{d} m_{i, j}}{\mathrm{~d} t}}{\frac{\partial p}{\partial T}}
\end{aligned}
$$

The basic (temperature) part of the thermal state equation, e.g., for the static enthalpy $\left.h_{i d}=\mathrm{f}\left(T,{ }_{,} m\right\}\right)$, yields with the equation (6) the relation between enthalpy and thermodynamic state quantities (pressure, temperature, etc.)

$$
\begin{aligned}
& H_{j}=H\left(T, p,\left\{{ }_{\mathrm{s}} m\right\}\right) \\
\frac{\mathrm{d} H_{j}}{\mathrm{~d} t}= & \frac{\partial H}{\mathrm{~d} T} \cdot \frac{\mathrm{d} T_{j}}{\mathrm{~d} t}+\frac{\partial H}{\mathrm{~d} p} \cdot \frac{\mathrm{d} p_{j}}{\mathrm{~d} t}+\sum_{i}^{s} \frac{\partial H}{\mathrm{~d} m_{i}} \cdot \frac{\mathrm{d} m_{i, j}}{\mathrm{~d} t}= \\
= & \frac{\partial H}{\frac{\mathrm{d} T}{\partial T}} \cdot\left(\frac{\mathrm{d} p_{j}}{\mathrm{~d} t}-\frac{\partial p}{\partial V} \cdot \frac{\mathrm{d} V_{j}}{\mathrm{~d} t}-\sum_{i}^{s} \frac{\partial p}{\partial m_{i}} \cdot \frac{\mathrm{d} m_{i, j}}{\mathrm{~d} t}\right)+ \\
+ & \frac{\partial H}{\mathrm{~d} p} \cdot \frac{\mathrm{d} p_{j}}{\mathrm{~d} t}+\sum_{i}^{s} \frac{\partial H}{\mathrm{~d} m_{i}} \cdot \frac{\mathrm{d} m_{i, j}}{\mathrm{~d} t}
\end{aligned}
$$

The thermal effects of chemical reactions are involved automatically if enthalpy considers reaction terms for combustible components (see the first term in the second row of the equation (8), in which chemical changes may be involved).

Versatile formulation of energy conservation uses total energy (internal + kinetic $\mathrm{K}+$ turbulent kinetic $k$ ones) or total enthalpy. Then, using the differential equation of state (8), the set of ODEs for an arbitrary number of FVs is solvable.

The energy conservation yields thus for the static enthalpy

$$
\begin{aligned}
& \frac{\mathrm{d} H_{j}}{\mathrm{~d} t}=\sum_{i=N_{j}}\left[\dot{V}_{j, i} \cdot\left(\frac{H_{K i} \cdot \gamma_{j, i}}{V_{i}}+\frac{H_{K j} \cdot \delta_{j, i}}{V_{j}}\right)+\alpha_{i, j} \cdot A_{i, j}\left(T_{i}-T_{j}\right)\right]+ \\
& +\sum_{k} \dot{Q}_{k, j}+V_{j} \cdot \frac{\mathrm{d} p_{j}}{\mathrm{~d} t}-m_{j}\left(\frac{\mathrm{d} K_{j}}{\mathrm{~d} t}+\frac{\mathrm{d} k_{j}}{\mathrm{~d} t}\right)-\frac{\mathrm{d} m_{j}}{\mathrm{~d} t} \cdot\left(K_{j}+k_{j}\right) .
\end{aligned}
$$

The thermodynamic state quantities (pressure, temperature, etc.) are evaluated iteratively combining the equations (8), (9) in the case of a mixture of real gases. Heat transfer to neighbouring FVs is taken into consideration by Newton's equation and by the terms $\boldsymbol{Q}_{\boldsymbol{k}}$ if necessary; turbulent kinetic energy $k$ may often be neglected at the current level of precision, and in the case of a big flow cross-section the same is valid for $K$.

Nevertheless, for use in association with experiments where pressure measurements are often the single output or for multi-zone models with equal pressure and different temperature inside FVs, it is better to write energy conservation in a form that provides the pressure derivative explicitly. It is possible in the case of ideal gas. The equation has to be supplemented by closures concerning heat release (reaction velocities) and heat transfer. Corrective heat flux $Q_{\text {corr }}$ (e.g., for heat radiation) and enthalpy corrective flux $H_{\text {corr }}$ may be used if necessary. Their meaning is important as corrections in inverse algorithms. Then energy conservation yields

$$
\begin{aligned}
& \frac{V_{j}}{\mathrm{\kappa}-1} \cdot \frac{\mathrm{d} p_{j}}{\mathrm{~d} t}= \\
& =\sum_{i=N_{j}}\left[\dot{V}_{j, i} \cdot\left(\rho_{i} \cdot h_{K, i} \cdot \gamma_{j, i}+\rho_{j} \cdot h_{K, j} \cdot \delta_{j, i}\right)+\alpha_{i, j} \cdot A_{i, j}\left(T_{i}-T_{j}\right)\right]+ \\
& -\frac{\kappa_{j}}{\kappa_{j}-1} \cdot p_{j} \cdot \frac{\mathrm{d} V_{j}}{\mathrm{~d} t}-\left(\{\mathrm{s} h\}^{T}-\frac{\kappa_{j}}{\kappa_{j}-1} \cdot T_{j} \cdot\left\{{ }_{\mathrm{s}} r\right\}^{T}\right) \cdot \frac{\mathrm{d}\{\mathrm{s} m\}_{j}}{\mathrm{~d} t}+ \\
& -m_{j} \cdot\left(\frac{\mathrm{d} K_{j}}{\mathrm{~d} t}+\frac{\mathrm{d} k_{j}}{\mathrm{~d} t}\right)-\frac{\mathrm{d} m_{j}}{\mathrm{~d} t} \cdot\left(K_{j}+k_{j}\right)+\left(\dot{Q}_{\text {corr }}+\dot{H}_{\text {corr }}\right) .
\end{aligned}
$$

In the case of small cross-sections and big velocities, the momentum conservation has to be considered. This vector equation can be written for a concrete coordinate system in the form of several equations, the number of which respects the number of coordinates. Thus in the case of Cartesian coordinates ( $\tau$ stands for friction losses at walls, and in this case another closure equation is needed for them, e.g., Stokes's and Reynolds's hypotheses, and a turbulence model):

$$
\begin{aligned}
m_{j} \cdot \frac{\mathrm{d} w_{j}}{\mathrm{~d} t}= & \sum_{i=N_{j}}\left[V_{j, i} \cdot\left(w_{i} \cdot \rho_{i} \cdot \gamma_{j, i}+w_{j} \cdot \rho_{j} \cdot \delta_{j, i}\right)+\right. \\
& \left.-p_{i} \cdot A_{i, j}-\tau_{i, j} \cdot A_{v i, j}\right]-w_{j} \cdot \frac{\mathrm{d} m_{j}}{\mathrm{~d} t}
\end{aligned}
$$

It is worth mentioning that this is not necessary for 1-D problems when velocities are low, unlike in 2 and 3-D cases, where energy and mass conservation does not give a complete set of equations more and the momentum conservation has to be used in any case. In cases of curvilinear coordinates appropriate inertia terms must be amended.

\section{Modularised structure of the code}

The general FV CFD methods have thus been re-applied for the 1-D or 0-D engine models. Unlike in full CFD approaches, the conditions at FVs boundaries must be described by 1-D gas dynamics expressions. The description of large engine structures comes into these models by connection through convective terms, describing the engine structure by means of the structure matrix of the whole simulated system. Closures may be involved in this open system according to applicator needs. They can be supplemented by inertia corrective terms, but in this case iterative solution of ODE with implicitly involved derivatives is needed. Because of the danger of stiffness implicit, variable-step or even iterative ODE solvers have to be applied. In the latter case there is no problem to implement inertia corrections. Code subroutines for ODE right-hand sides have been programmed as an open system to which closures may be implemented. Function tests for some typical examples were done. Other implementations and - based on them - automatic structuring of the source code applied to a concrete system will be done in the future.

\section{Model calibration based on experiments}

The concept of procedures inverted to the described analytical algorithms is used for engine experiment evaluation (rate-of-heat-release, $\mathrm{NO}_{\mathrm{x}}$ formation, valve aerodynamic parameters, turbine efficiency, etc.). The main advantage of well-structured transparent algorithms for thermodynamic 
calculations is the fact that they can be inverted and a measured quantity pattern can be used for computing other values (in most cases the pressure traces inside a cylinder or manifold create source data).

Concerning rate-of-heat-release $(\boldsymbol{R O H R})$, the aim is to find the main reaction component of fuel combustion, i.e., one of the components $\left.\mathrm{d}\left\{{ }_{\mathrm{s}} m\right\} / \mathrm{d} t\right)$ in a certain reaction. The other $\mathrm{d}\{m\} / \mathrm{d} t$ for the chosen reaction are coupled by stoichiometry, using appropriate column only of the $\left\|_{\mathrm{s}}{ }_{\mathrm{r}} C\right\|$ matrix [9], i.e., the transformation vector $\left\{_{s ; r}=\right.$ main $\left.C\right\}$ of the chosen reaction is used. Other (side) reactions are calculated according to the model chosen. Absolute values of correction terms $Q_{\text {corr }}$ and/or $H_{\text {corr }}=m * h$ in the pressure differential equation should be minimised if the main reaction component is correct and other influence (i.e., leakage) is small. This procedure is typical for the $\boldsymbol{R O H R}$ computations used to calibrate model closures (see the example in Fig. 5). After it, the enthalpy correction term may thus be distributed to the product of the known specific in-cylinder enthalpy and an unknown mass flux caused by a piston-ring leakage. Then it can be used during combustion as a correction.

In other cases, e.g., during charge exchange, this term is useful in obtaining the essential mass flux in valves. The flow through a single set of valves and the appropriate discharge coefficient may be checked during pure inlet or exhaust. Another way is to substitute the complete pressure equation by the measured pressure and to calculate only the rest of the system described by the other equations. This procedure can be used to analyse more complicated problems (e.g., pre-chamber combustion where mass flux and heat release are coupled) to avoid amplification of errors caused by an imperfect part of the system model.

The examples of the $\boldsymbol{R O H R}$ traces evaluated from experiments at a natural-gas, spark-ignition engine are shown in Fig. 5. They create a suitable base for the extrapolation of engine parameters during a design optimisation. Some supplementary measured data (fuel and air flow rates) and amending assumptions concerning the temperature at the start of compression had to be used during the evaluation. It is important that these supplementary conditions are again based on the same equations during the both evaluation and simulation processes.

\section{Conclusions}

An efficient way to maximum use of internal combustion engine potential involves a combination of thermodynamic analysis and engine gear modelling starting with an idealised cycle in the case of configuration design. The computerised entropy diagram provides an initial procedure of the thermodynamic optimisation. The advanced methods (1-D, CFD) must be properly structured and equipped by suitable interfaces, which enables the user to describe a complicated engine structure. Nevertheless, even these advanced methods contain always some empirical closure equations. Methods for model calibration based on experiments are therefore essential and useful tools, if the differential equations of the simulation model are used an solved by inverted algorithm.

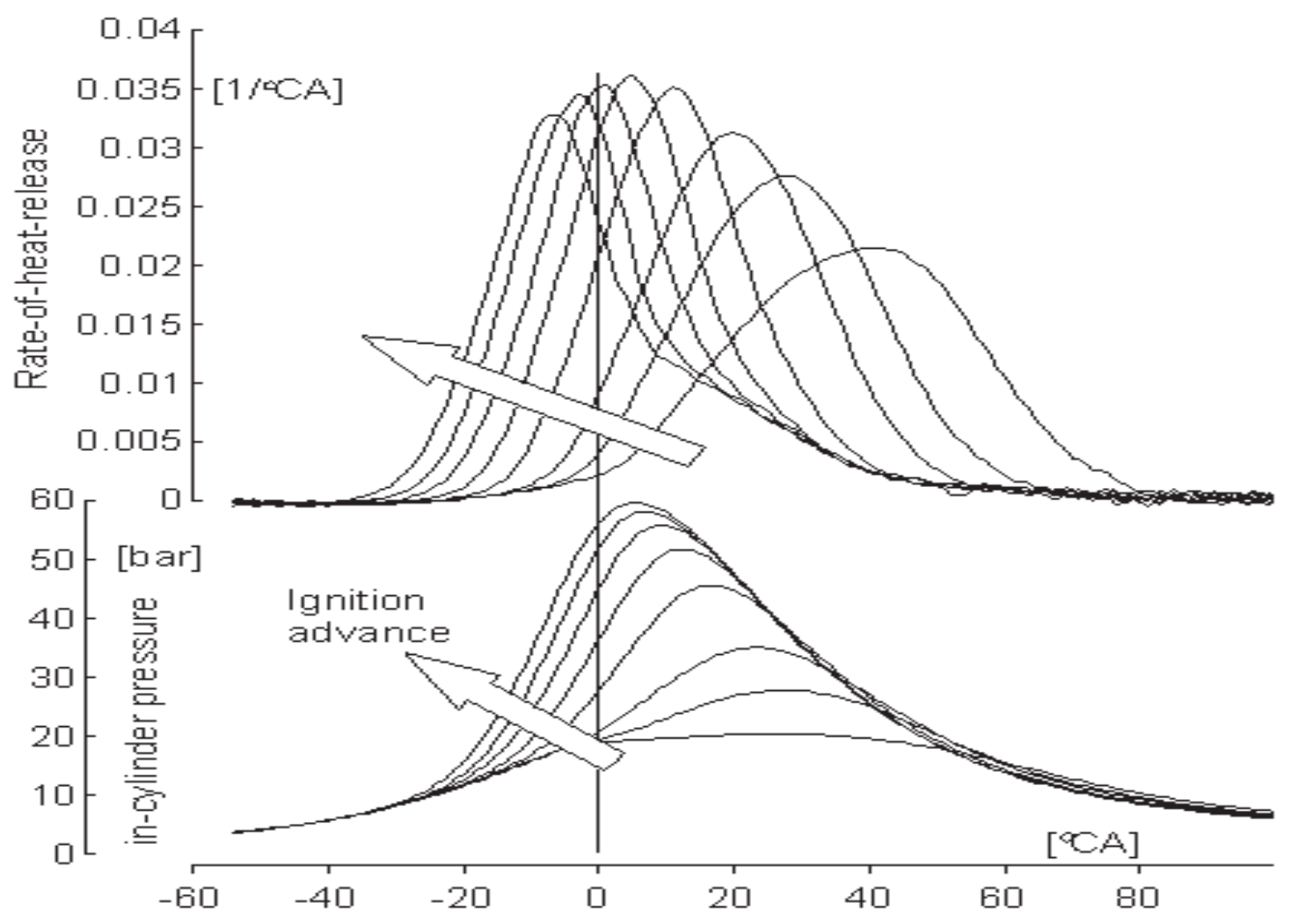

Fig. 5: Evaluation of in-cylinder pressure records for a spark ignition, natural gas fuelled engine at different ignition advance using a measured pressure signal, measured mean mixture flow rate and supplemented by simulated temperature at the start of compression 
These models are presented here, creating a hierarchical structure from the simple algebraic model, providing transparent and instructive results, to the most complicated models, which can be used for final optimisation. The modular structure of the latter models enables an appropriate model of unconventional engine lay-out to be constructed.

\section{Acknowledgements}

This research has been supported by Research Project MSM 212200008 of the Ministry of Education, Czech Republic.

\section{References}

[1] Macek, J., Valášek, M.: Initial Methodology for Concurrent Engineering. In: "Proc. of First International Conference on Advanced Engineering Design" (Editors: Fiala, P., Smrček, L.), Prague, ČVUT 1999, pp. 286-290, ISBN 80-01-02055-X

[2] Ciesla, Ch., Keribar, R., Morel, T.: Engine/Powertrain/Vehicle Modeling Tool Aplicable to All Stages of the Design Process. SAE Paper 2001 (in review)

[3] Macek, J., Steiner, T.: Advanced Multizone Multidimensional Models of Engine Thermoaerodynamics. In: " $21^{\text {st }}$ Intl. Congress on Combustion Engines” (Editor M.K.Eberle), London, CIMAC. 1995, pp. D10/1-D10/18

[4] Macek, J., Polášek, M.: Advanced Eulerian Multizone Model - Versatile Tool in Moveable Boundary Problem Modeling. In: " 8 th International Symposium on Computational Fluid Dynamics", Proceedings on CD-ROM. Bremen, ZARM 1999, pp. 1-20

[5] Durst, F., Weclas, M.: A new type of Internal Combustion engine based on the porous medium combustion technique. Journal of Automobile Engineering 2001 (in press)

[6] Macek, J.: Compression or Heat Regeneration in the Cycle of Reciprocating Engine - New Results of Old Methods. In: “KOKA 2000" (Editor Hlavňa, V.), Žilina, Technical
University of Žilina, 2000, pp. 117-122, ISBN 80-7100-736-6

[7] Macek, J.: A Simplified Model of a Valve Gear with Friction. In: "Sborník z XXX. mezinárodní konference" (editors Macek, J., Baumruk, P.), Praha, ČVUT 1999, pp. 17-27, ISBN 80-01-01972-1

[8] Macek, J., Remek, B.: Simplified Model of Losses in Crank Gear. In: "MOTORSYMPO '99" (Editors: Macek, J., Kroebl, L.), Praha, ČVUT, FSI, 1999, pp. 11-21, ISBN 80-01-01985-3

[9] Macek, J., Kozel, K., Baumruk, P., Takáts, M., Hatschbach, P., Bendl, F.: Computational Fluid Dynamics or Zone Models - Pros and Cons in IC Engine Research. In: "World Automotive Congress of FISITA 1996" (Edts. M. Apetaur, M. Hanke) Praha, ČSAT, 1996, Technical Papers on CD ROM, pap. P14.02, pp. 28

[10] Macek, J., Polášek, M.: General Method for Modeling of Quasi-1D Subsonic Flows and Its Application in CFD Boundary Conditions. In: "Topical Problems of Fluid Mechanics 2000" (Editors: Kozel, K., Př́íhoda, M.), Prague, Academy of Science of the Czech Republic, Institute of Thermomechanics, 2000, pp. 57-60, ISBN 80-85918-55-2

Prof. Ing. Jan Macek, DrSc. phone: +420224352504 fax: +420224352500 e-mail:macek@fsid.cvut.cz

Doc. Ing. Michal Takáts, CSc. phone: +420220395127 fax: +420224352500 e-mail: takats@fsij.fsid.cvut.cz Josef Božek Research Centre Czech Technical University in Prague Faculty of Mechanical Engineering Technická 4, 16607 Praha 6, Czech Republic 